\title{
Rotationally supported Virgo dwarf elliptical galaxies
}

\author{
L. van Zee ${ }^{1}$, E.D. Skillman ${ }^{2}$ and M.P. Haynes ${ }^{3}$ \\ ${ }^{1}$ Department of Astronomy, Indiana University, Bloomington, IN 47405, USA \\ email: vanzee@astro.indiana.edu \\ ${ }^{2}$ Astronomy Department, University of Minnesota, Minneapolis, MN 55455, USA \\ email: skillman@astro.umn.edu \\ ${ }^{3}$ Center for Radiophysics and Space Research and National Astronomy and Ionosphere Center, \\ Cornell University, Ithaca, NY 14853, USA \\ email: haynes@astro.cornell.edu
}

\begin{abstract}
We present the results of optical stellar rotation curves of 16 dwarf elliptical galaxies in the Virgo Cluster. Approximately half of this sample have significant velocity gradients along their optical major axis, with typical rotation amplitudes of $20-30 \mathrm{~km} \mathrm{~s}^{-1}$. Evidence for a relationship between the rotation amplitude and galaxy luminosity is found and agrees well with the Tully-Fisher relation. The similarity in the scaling relations of dIs and dEs implies that it is unlikely that dEs evolve from significantly more luminous galaxies. These observations reaffirm the possibility that some cluster dwarf elliptical galaxies may be formed when the neutral gaseous medium is stripped from dwarf irregular galaxies in the cluster environment.
\end{abstract}

Keywords. galaxies: clusters: individual (Virgo), galaxies: dwarf, galaxies: evolution, galaxies: kinematics and dynamics

\section{Introduction}

One of the intriguing aspects of the dwarf galaxy class is the apparent similarity between gas-rich and gas-poor dwarf galaxies. Unlike their giant cousins, both star forming and non-star forming dwarf galaxies have exponential light distributions and share a similar range of size, surface brightness, and luminosity (e.g., Binggeli 1994). A multitude of evolutionary scenarios have been proposed to explain the relationship between gas-poor dwarf elliptical galaxies and gas-rich dwarf irregular galaxies (e.g., Dekel \& Silk 1986, Silk et al. 1987, Davies \& Phillipps 1988). The key component of these models is the gas removal process: gas is removed either through a "blow-out" following a starburst episode, or via ram pressure stripping as the galaxy encounters the hot intergalactic medium (IGM) or intracluster medium (ICM). Interestingly, if either of the above scenarios are correct, the stellar remnant $(\mathrm{dE})$ should have the same kinematic properties as the progenitor $(\mathrm{dI})$.

However, the ground-breaking kinematic work by Bender \& Nieto (1990) and Bender et al. (1991) suggested that dEs and dIs may not have similar kinematic properties: of the 5 dwarf elliptical galaxies in these two early studies, none are supported by rotation $\left(\mathrm{V}_{\text {rot }}<20 \mathrm{~km} \mathrm{~s}^{-1}\right)$; in contrast, most dwarf irregular galaxies of comparable mass (or luminosity) are rotationally supported. The number of dwarf elliptical galaxies with spatially resolved rotation curves is still quite small, however. Stellar kinematics of large samples of dwarf elliptical galaxies are needed to investigate fully kinematic constraints on evolutionary scenarios. Here, we summarize the results from a program to measure the stellar kinematics of sixteen dwarf elliptical galaxies in the Virgo Cluster. 


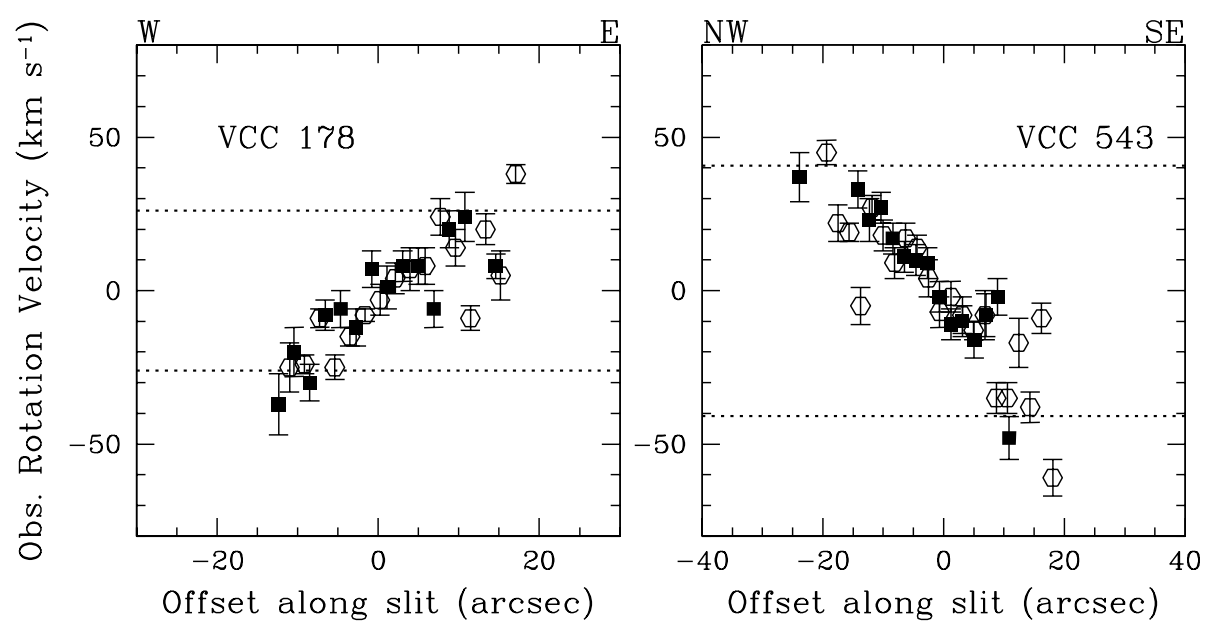

Figure 1. Example rotation curves derived from the Mg Ib (open circles) and Ca triplet (filled squares) lines. The dashed lines indicate the maximum velocity width measured from the observed rotation curve.

\section{Sample Selection and Observations}

The target galaxies were selected from the Virgo Cluster Catalog (Binggeli et al. 1985) based on their apparent magnitude $\left(\mathrm{m}_{\mathrm{B}}<15.5\right)$, morphological classification $(\mathrm{dE})$, and on their apparent ellipticity $(\epsilon>0.25)$. The latter criterion was imposed to mitigate the affects of disk projection in the measurement of rotational velocities. The sample was further restricted to the luminous dwarf elliptical galaxies in the VCC with measured recessional velocities that place them within the Virgo Cluster. The targeted galaxies were selected to include a range of environments within the cluster, sampling both the cluster core and the outer field.

High resolution optical spectra were obtained with the Double Spectrograph on the $5 \mathrm{~m}$ Palomar telescope. Full details of the observing parameters are described in van Zee, Skillman, \& Haynes (2004). Briefly, the blue side of the spectrograph was centered at the $\mathrm{Mg}$ Ib triplet, with wavelength coverage of $4800-5700 \AA$ and an effective resolution of $2.3 \AA(0.88 \AA /$ pix $)$ while the red side was centered at the Ca triplet, with wavelength coverage of $8250-8900 \AA$ and an effective resolution of $1.6 \AA(0.64 \AA /$ pix $)$. Due to the low surface brightness nature of the $\mathrm{dE}$ galaxies, long integration times (on the order of 2 - 3 hours per pointing) were necessary to have sufficient signal-to-noise in the absorption lines to trace the stellar kinematics to large radii.

\section{Results}

Rotation curves for two of the 16 galaxies in this sample are shown in Figure 1. Seven of the dwarf elliptical galaxies in this sample are dominated by rotation. Furthermore, as illustrated by Figure 2, the correlation between rotation width (corrected for inclination) and absolute magnitude for the dwarf elliptical galaxies shows a remarkable agreement with the Tully-Fisher relation. Such a correlation is expected if the dwarf elliptical galaxies in this sample are the end products of dwarf irregular galaxies which have been stripped of their ISM. 


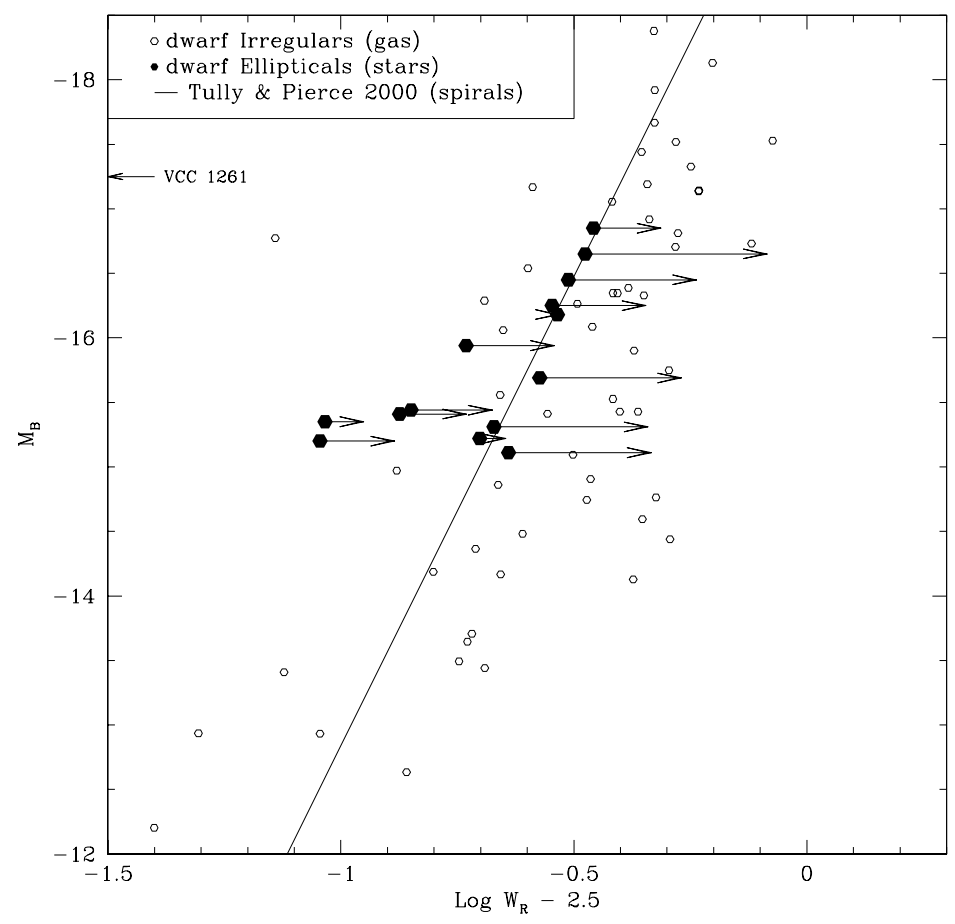

Figure 2. Tully Fisher relation for dIs, dEs, and spirals. The dE galaxies are shown as both filled hexagons (observed maximum rotation width) and as arrows (maximum rotation width assuming the rotation curve could be traced to 2.45 scale lengths). The open circles are the dwarf irregular galaxies compiled in van Zee (2001). The straight line shows the expected relationship for gas-rich spiral galaxies (Tully \& Pierce 2000).

These results are significantly different than those reported by Geha et al. (2002, 2003) and the early studies of Bender \& Nieto (1990) and Bender et al. (1991) where it appeared that dwarf elliptical galaxies had no significant rotation component. However, even though these results are counter to previous observations, they should not have been surprising. It is well known that galaxies located near the center of the Virgo cluster are deficient in neutral hydrogen relative to their counterparts in the field (e.g., Giovanelli \& Haynes 1983, Solanes et al. 2001). For giant spiral galaxies, the loss of their ISM may result in modest morphological changes, including suppression of their star formation activity (e.g., Koopmann \& Kenney 1998). However, despite these changes, gas-deficient giant spiral galaxies are likely still to be classified as spiral galaxies since there are striking morphological differences between giant spiral and elliptical galaxies. This is not the case for dwarf galaxies. Dwarf elliptical galaxies and dwarf irregular galaxies share common morphological parameters; both have approximately exponential surface brightness profiles and have similar central surface brightnesses and scale lengths. Thus, their structural parameters do not provide a strong morphological distinction between dwarf elliptical galaxies and dwarf irregular galaxies; rather, their morphological classification is highly dependent on the patchy appearance (or absence thereof) due to irregular star formation activity across the surface of the galaxy. If star formation ceases in a dwarf galaxy, the resulting system is likely to be classified as a dwarf elliptical galaxy once the massive stars, which provide the patchy contrast, have evolved off of the main sequence. 
In fact, it is this morphological commonality that first led to the idea that dwarf elliptical galaxies could evolve passively from dwarf irregular galaxies after a starburst episode.

\section{Conclusions}

The kinematic observations presented here indicate a remarkable similarity between the kinematic properties of many dwarf elliptical and dwarf irregular galaxies; these results re-open the possibility that some dEs are formed from passive evolution of dIs. To some degree, the question of whether dwarf elliptical galaxies evolve from dwarf irregular galaxies is tautological since every $\mathrm{dE}$ must have been gas-rich and star-forming at some point in the past. Standard morphological classification would identify these gas-rich, low mass galaxies as dIs (see also Skillman's contribution to these proceedings). Thus, the relevant question is not whether a $\mathrm{dE}$ evolves from a $\mathrm{dI}$, but what caused the progenitors of dEs to stop forming stars and to lose their ISM.

A multitude of evolutionary scenarios are possible for cluster dwarf elliptical galaxies. Since dwarf irregular galaxies are dark matter dominated, removal of their ISM will have only a modest effect on their kinematics. However, subsequent passages through the cluster could further disrupt the stellar kinematics via galaxy harassment or merging events (e.g., Mayer et al. 2001a,b). Thus, if dIs are converted into dEs via ram pressure stripping, one might expect a correlation between the location of dwarf elliptical galaxies with significant rotation and the cluster center. While the present sample is relatively small, there is a slight hint of such a relationship, in the sense that galaxies with littleto-no rotation appear to be located predominantly in the cluster core or in high density clumps, while those with significant rotation are in the outskirts of the cluster. This is consistent with the idea that gas-rich dwarf galaxies are stripped of their gas during a passage through the intracluster medium; the non-rotating dwarf elliptical galaxies may be remnants of gas-rich systems that have made multiple passes through the ICM, or had a catastrophic event occur. While these results are suggestive, additional knowledge of the star formation histories and environmental influences of typical cluster dEs are highly desirable to enable further insight into the evolution of dwarf elliptical galaxies.

\section{Acknowledgements}

This work was supported in part by the Herzberg Institute of Astrophysics and Indiana University.

\section{References}

Bender, R. \& Nieto, J.-L. 1990, A\&A 239, 97

Bender, R., Paquet, A. \& Nieto, J.-L. 1991, A\&A 246, 349

Binggeli, B. 1994, in Meylan, G., Prugniel, P., eds ESO/OHP Workshop on Dwarf Galaxies ESO, Garching, p. 13.

Binggeli, B., Sandage, A. \& Tammann, G.A. 1985, AJ 90, 1681

Davies, J.I. \& Phillipps, S. 1988, MNRAS 233, 553

Dekel, A. \& Silk, J. 1986, ApJ 303, 39

Geha, M., Guhathakurta, P. \& van der Marel, R.P. 2002, AJ 124, 3073

Geha, M., Guhathakurta, P. \& van der Marel, R. P. 2003, AJ 126, 1794

Giovanelli, R. \& Haynes, M.P. 1983, AJ 88, 881

Koopmann, R.A. \& Kenney, J.D.P. 1998, ApJ 497, L75

Mayer, L., et al. 2001a, ApJ 547, L123

Mayer, L., et al. 2001b, ApJ 559, 754

Silk, J., Wyse, R.F.G. \& Shields, G.A. 1987, ApJ 322, L59 
Solanes, J.-M., et al. 2001, ApJ 548, 97

Tully, R.B. \& Pierce, M.J. 2000, ApJ 533, 744

van Zee, L. 2001, AJ 121, 2003

van Zee, L., Skillman, E.D. \& Haynes, M.P. 2004, AJ 128, 121

\section{Discussion}

CONSELICE: The fact that the dEs in the center of Virgo do not tend to be the rotating systems, is it possible that dynamic effects are halting the rotation, or is another $\mathrm{dE}$ formation scenario necessary?

VAN ZEE:: We expect tidal stirring to also change the kinematics of some dEs. One thing to keep in mind, however, is that the "non-rotating" dEs have only been observed along the major axis; if they are tumbling, or if we are looking at face-on systems $(\epsilon<0.1)$, we would not expect to see a velocity gradient with the data in hand.

Gallagher:: Have you considered the possibility that a wider range of galaxy types (somewhat more massive than Irrs) could feed into the present day dEs? For example, a galaxy falling in at an earlier epoch and being stripped would have a much lower surface brightness (more extended stellar pop and fewer stars), and so we might, e.g., get M33-types into rotating dwarfs?

VAN ZEE:: There are two observations that indicate that the progenitor population is not bright galaxies that have been disrupted. First, the rotation curve data is inconsistent with the velocity widths expected for giant spirals. Second, the observed metallicities are low, similar to the abundances measured in dwarf irregular galaxies at similar luminosity.

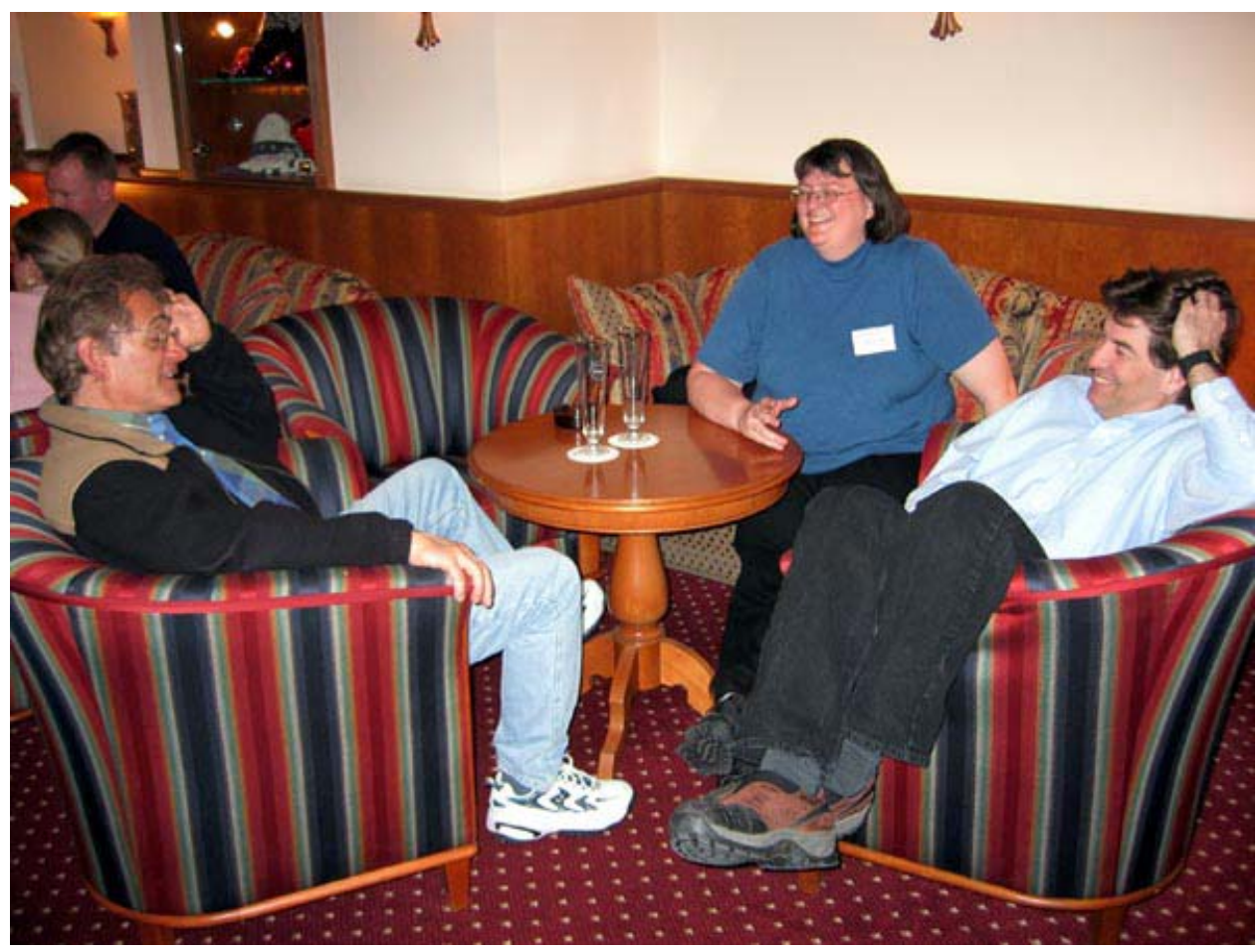

